\title{
Wet, volatile, and dry biomarkers of exercise-induced muscle fatigue
}

\author{
Josef Finsterer $^{{ }^{*}}$ and Vivian E. Drory ${ }^{2}$
}

\begin{abstract}
Background: The physiological background of exercise-induced muscle fatigue(EIMUF) is only poorly understood. Thus, monitoring of EIMUF by a single or multiple biomarkers(BMs) is under debate. After a systematic literature review 91 papers were included.

Results: EIMUF is mainly due to depletion of substrates, increased oxidative stress, muscle membrane depolarisation following potassium depletion, muscle hyperthermia, muscle damage, impaired oxygen supply to the muscle, activation of an inflammatory response, or impaired calcium-handling. Dehydration, hyperammonemia, mitochondrial biogenesis, and genetic responses are also discussed. Since EIMUF is dependent on age, sex, degree of fatigue, type, intensity, and duration of exercise, energy supply during exercise, climate, training status (physical fitness), and health status, BMs currently available for monitoring EIMUF have limited reliability. Generally, wet, volatile, and dry BMs are differentiated. Among dry BMs of EIMUF the most promising include power output measures, electrophysiological measures, cardiologic measures, and questionnaires. Among wet BMs of EIMUF those most applicable include markers of ATP-metabolism, of oxidative stress, muscle damage, and inflammation. $\mathrm{VO}_{2}$-kinetics are used as a volatile BM.

Conclusions: Though the physiology of EIMUF remains to be fully elucidated, some promising BMs have been recently introduced, which together with other BMs, could be useful in monitoring EIMUF. The combination of biomarkers seems to be more efficient than a single biomarker to monitor EIMUF. However, it is essential that efficacy, reliability, and applicability of each BM candidate is validated in appropriate studies.
\end{abstract}

Keywords: Biomarker, Biological marker, Muscle fatigue, Monitoring, Muscle exercise, Exercise fatigue, Exhaustion, Endurance

\section{Background}

A biomarker (BM) is a measurable molecule or test, to objectively monitor the change of a condition or process over time, after treatment, or after training [1]. BMs may be used for diagnostic purposes, for monitoring purposes, or for risk assessment [2]. BMs can be used as an indicator of a biological state, or to objectively assess a biological or pathological process. To assess the degree of muscle fatigue (continuous reduction of muscle force (maximal voluntary contraction (MVC)) during exercise (exercise-induced muscle fatigue (EIMUF), peripheral fatigue) and the impact of exercise on the skeletal muscle,

* Correspondence: fipaps@yahoo.de

'Krankenanstalt Rudolfstiftung, Postfach 20, 1180 Vienna, Austria

Full list of author information is available at the end of the article various BMs have been proposed but their reliability is questionable [3]. BMs of EIMUF need to be differentiated from BMs of physical fitness, muscle damage (injury), of overtraining, of inflammation, and from BMs of central fatigue (central nervous system disease) or fatigue due to systemic disease (anemia, menstruation, malignancy, cardiac disease, chronic infection, vitamin deficiency, or hepatopathy). To approach the topic of monitoring EIMUF it is essential to establish a definition of EIMUF and to elucidate its physiologic background. Aim of this review was to describe and discuss established findings and new aspects emerging from the clinical and experimental literature about BMs of EIMUF. These new aspects are expected to influence experimental modelling, development of diagnostics, and development of new BMs to more accurately monitor EIMUF. 


\section{Method}

Data for this review were identified by searches of MEDLINE, Current Contents, Springerlink, Wiley, EBSCO, Ovid, and Web of science by applying a sensitive search strategy using combinations of the following search terms: "biomarker", "biological marker", in combination with "fatigue", "fatigability", "muscle", "exercise", "exercise-induced", and "exhaustion". Further manual search was conducted to identify other relevant articles from cross-references. Randomized (blinded or open label) clinical trials, longitudinal studies, case series, and case reports were considered. Abstracts and reports from meetings and animal studies were not included. Only articles about humans and published in English, French, Spanish, or German between 1966 and 2015 were considered. Appropriate papers were studied and discussed for their suitability to be incorporated in this review.

\section{Results}

Altogether 134 papers were found to be suitable to match with the intentions of the review. Of these, 91 were selected, because they were available as full papers, they provided most recent data, they provided an extensive, in-depth discussion of their results, and they compared previous findings with the current results.

\section{Biomarkers \\ Requirements a BM must meet}

Not each molecule or test is eligible to serve as an appropriate BM of EIMUF. Requirements a BM must meet are that the correlation between the measurement and the represented process is linear, that it shows the continuous change of the process, and that it appropriately reflects the outcome of a condition in the sense of a linear outcome measure. Additionally, BMs must register quick changes (translational marker) and need to change as a function of the process being monitored. They need to be stable without appreciable diurnal variations, need to correlate significantly with exercise intensity, and need to be present in detectable amounts in easily accessible biological fluids [2]. It is also essential that the usefulness and efficiency of a BM has been validated and proven, not to unnecessarily increase costs. Generally, BMs need to be easily collected (easily accessible tissues), cheap, the test should be easy to perform with widely available equipment, should be reliable when tested by different examiners and repeatedly, and independent of age, sex, environmental and climate conditions, pre-existing training condition, food, hydration, daytime, and the exercise monitored. It is also important that the test carried out to monitor EIMUF does not further induce fatigue. Most of the available BMs of EIMUF do not meet these requirements [3].

\section{Dependency of a BM}

Since there is no ideal BM, BMs depend on various different factors. These include age, sex, degree of general fatigue, type, intensity, frequency, and duration of exercise (e.g. voluntary or electrical stimulation), type of contraction (isometric, isotonic, intermittent, continuous), environmental climatic conditions (temperature, humidity, wind speed), food, daytime. and physiological and training status (physical fitness) of an individual [1, 4]. A BM may also depend on the energy supply during exercise, on hydration, and on the health status (healthy or diseased individuals) [5]. The optimal choice of a BM is not only dependent on the factors just mentioned but also on how good the physiology of the process to be monitored is understood.

\section{Classification of BMs}

BMs can be classified according to the method applied as wet, volatile, or dry BM [6], as translational or nontranslational $\mathrm{BM}$, as invasive or non-invasive $\mathrm{BM}$, as validated or non-validated BM, or as BM for measuring focal or generalised EIMUF. Most wet BMs derive from blood, saliva, or urine. Only rarely other body fluids are investigated. Most dry BMs are measures of the power output, cardiac parameters, or scales or questionnaires. Needle and particularly surface electromyography (EMG) are also applied for monitoring EIMUF. The choice of a BM for monitoring EIMUF depends on the duration, type, and intensity of exercise performed. There is a difference if the exercise is short-lasting for only a few seconds or minutes or a long-lasting condition, like in marathon runners or triathloners. It is also strongly dependent on the type of contraction. BMs can be also categorised according to the exercise performed, which can be either assessed by another test or can be the BM itself (Fig. 1). BMs of EIMUF may be further differentiated into external and internal BMs. External load quantifying tools include power output measuring devices, time-motion analysis (to analyse movement patterns in team-sport), or movement kinematics or kinetics, whereas internal load quantifying tools include, for example, cardiac parameters, neuromuscular function, biochemical, hormonal, or immunological assessments, and questionnaires (Table 1) [3].

\section{Classification of exercise}

Exercise, carried out to induce fatigue, may be classified according to various criteria such as duration, intensity, or type of muscle contraction or according to the type of energy production (aerobic or anaerobic). According to duration and intensity, exercise may be classified as short-duration, low-intensity exercise, as short-duration, high intensity exercise, or as long-duration exercise. According to the type of energy production exercise may be classified as $<20 \mathrm{sec}$ in duration (up to $90 \%$ anaerobic 


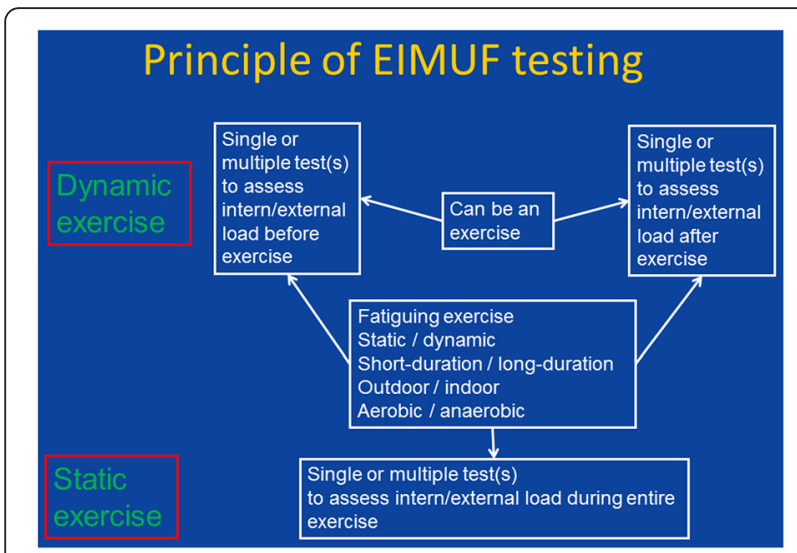

Fig. 1 General test arrangement to monitor EIMUF. Monitoring tests may be generally carried out before and after the test (usually for dynamic exercise) or continuously during the entire test (usually for static exercise)

energy), $20 \mathrm{sec}$ to $1 \mathrm{~min}$ (aerobic and anaerobic energy), and exercise lasting $>1$ min ( $>50 \%$ aerobic energy). It is essential to know that exercise may not induce fatigue if exercise is mild and carried out for a very short time. Exercise may be also classified as static (no movement of entire body) or as dynamic (movement of entire body (e.g. sport)) [7]. Exercise may be carried out within an experimental setting (e.g. standardised ergometer (simulated)) or as an open-air exercise (running, walking (real)) [8]. Muscle contraction during exercise may be voluntary or stimulated, continuous or discontinuous, or isometric (no change of length), isotonic (muscle length changes) which can be either eccentric or concentric, or isokinetic (like isotonic but with constant speed).

\section{Exercise-induced muscle fatigue (EIMUF)}

EIMUF is a complex and multimodal process involving various metabolic and functional mechanisms.

\section{Definition of EIMUF}

Due to the uncertainties about the complex nature of EIMUF a number of different definitions exist depending on the underlying experimental model or the conditions under which EIMUF develops [3]. One of the most commonly applied definitions of fatigue describes EIMUF as "a failure to maintain the required or expected muscle force" (reduced power output (work rate), ATP turnover rate) [9]. In this definition fatigue is regarded as decrement of power production (decline of muscle force, decreased force output) and EIMUF corresponds to the reduction of muscle force during exercise $[10,11]$. Another popular and more general definition of EIMUF defines it as "inability to complete a task that was once achievable within a recent time frame" [3]. Exercise scientists define EIMUF as
Table 1 BMs of EIMUF currently under discussion

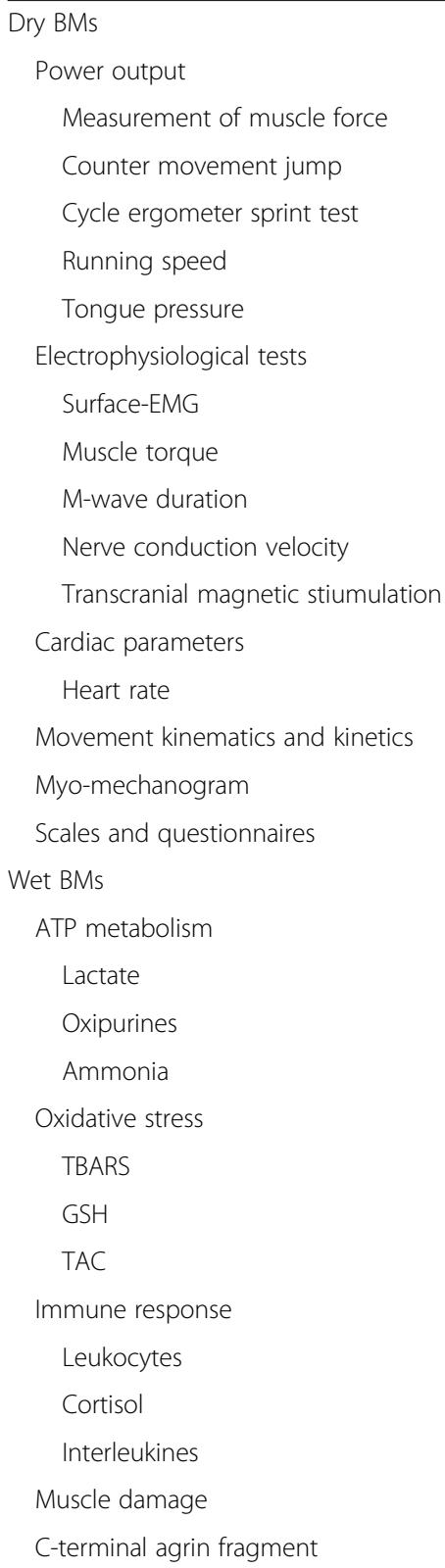

Volatile BMs

"exercise-induced impairment of muscle performance" [10]. Exercise intensity is initially sustained by recruitment of new motor units and help from synergistic muscles [10]. As exercise proceeds, technique and motor skill execution deviates to maintain outcomes but lastly they deteriorate resulting in reduced accuracy and velocity [10]. EIMUF may be also defined as divergence between external load (work completed by a subject and measured independently of its internal characteristics) and internal load (relative physiological or psychological stress imposed) [3]. A combination of both may be 
important for monitoring EIMUF [3]. An example for an external load is the power output sustained for a given duration. Examples for internal loads are the heart rate or perception of the effort [3]. Compensatory mechanisms of fatigue are an altered CNS muscle recruitment pattern, recruitment of additional motor units, synchronisation of firing frequencies, and increase of firing frequencies. Fatigue may be physiologic or pathologic. It may last for a short while or for days (e.g. 6 days in soccer players after a match) [12]. Fatigue is dependent of similar factors as described for BMs [3].

\section{Physiology of muscle fatigue}

To understand and monitor EIMUF it is essential to include all physiological aspects of EIMUF. Currently, however, the physiology of EIMUF is not fully understood because it is difficult to investigate an active muscle and because several processes run off simultaneously during any type of muscle exercise [13]. Thus, it is essential for future studies to find out which changes and processes contribute most to EIMUF. Presumably responsible for EIMUF are a shortage of substrates to maintain contraction, such as carbohydrates, calcium, sodium, potassium, or ATP, continuous oxidative stress, electrophysiological changes due to decrease of membrane depolarisation, hyperthermia, muscle damage, inflammatory processes, calcium-handling, and impaired oxygen supply (hypoxia) of the contracting muscle $[14,15]$.

Depletion of carbohydrate deposits During a marathon there is progressive depletion of carbohydrate reservoirs (glucose deposits in the liver and muscle) due to the active muscle $[14,16]$. Insufficient supply of carbohydrates during a marathon can lead to hypoglycemia and consecutively muscle fatigue [17]. In addition to carbohydrates other essential metabolites, such as ascorbic acid, calcium, sodium, potassium, and ATP may be depleted during exercise [7]. Depletion of ATP may be associated with an increase in ADP, which inhibits sodium/potassium ATPases and calcium-ATPases [18].

Production of reactive oxygen species (ROS) Exercise induces excessive production of ROS leading to oxidative stress, fatigue and reduced exercise performance $[19,20]$. Oxidative stress is highest immediately after exercise but may rise again hours after finishing exercise [2]. Accordingly, oxidative stress parameters are typically increased immediately after exercise [21]. Oxidative stress in form of ROS is implicated in the damage of various macromolecules, immune dysfunction, and muscle damage [19]. Oxidative stress may not only be responsible for EIMUF but also for impaired recovery from exercise [22].
Membrane depolarisation due to potassium depletion During high-intensity, long-term exercise potassium is continuously leaking from the muscle cell decreasing the membrane potential and thus depolarising the muscle membrane, therefore currents cannot propagate along the membrane or get into the triad [23]. Consecutively, the muscle becomes weak [1,24]. Additionally, depletion of potassium from muscle cells results in progressive hyperkalemia with increasing exercise intensity [25].

Hyperthermia Particularly after long-duration exercise hyperthermia (exertional heat illness, core temperature $>39^{\circ}$ ) develops, reducing muscle performance [14, 21]. Interestingly, those with high post-exercise core temperature maintain a steady pace throughout the exercise while those with a lower post-exercise core temperature show lower pace at the end of the run [14]. Thus, post-race temperature correlates positively with mean running pace [14]. This could be due to increased muscle damage among those with low pace and thus lower core temperature [14].

Muscle damage Muscle damage is another factor contributing to muscle fatigue during endurance activity. In a study of 25 triathlon participants there was a positive correlation between reduced muscle performance and blood markers of muscle damage, such as creatine-kinase (CK), lactate-dehydrogenase (LDH), or myoglobin [26]. This study further showed that there is a relation between muscle damage and fatigue [26]. Accordingly, marathoners with higher levels of running fatigue, develop higher levels of $\mathrm{CK}, \mathrm{LDH}$, and myoglobin compared to marathoners with low levels of running fatigue [14]. Another study found a positive correlation between post-race urinary myoglobin concentration, an indirect marker of muscle damage, and the decrease of muscle performance after a marathon [27]. When performing muscle biopsy before and after a marathon, it has been evidenced that a marathon produces muscle fiber necrosis and inflammation [28]. These morphological alterations are similar to those found in rhabdomyolysis [28]. These findings suggest that muscle fatigue, particularly during endurance exercise, may partly derive from muscle damage. Muscle damage is one of the most relevant sources of EIMUF in long-distance runners [26].

Cardiac stress Cardiac stress as measured by the cardiac stress index (CSI), using a non-linear, detrended fluctuation analysis (DFA) of the heart rate, was positively correlated with the Borg rating perceived exertion (RPE) scale suggesting that cardiac stress contributes to EIMUF [29]. Since the CSI effectively quantifies the degree of cardiac stress during exercise, it can be used to monitor the cardiac condition during physical exercise [29]. Additionally, it has been 
shown in Sprague-Dawley rats that heart failure speeds up the microvascular $\mathrm{pO}_{2}$ mean response time (blood-muscle $\mathrm{O}_{2}$ driving gradient) [30]. Conversely, in the soleus muscle of rats low microvascular $\mathrm{pO}_{2}$ reduces the blood-muscle $\mathrm{O}_{2}$ driving gradient [30].

Inflammation Inflammatory processes are induced during exercise as a result of muscle metabolism and muscle damage [22]. Muscle biopsy from participants after a marathon showed muscle fiber necrosis and inflammation [28]. Classical BMs of inflammation are the blood leukocytes, C-reactive protein, interleukines, and serum or salivary cortisol [22].

Calcium handling There are some indications that impaired calcium handling contributes to the development of EIMUF [31]. Sarcalumenin is a Ca-binding protein of the sarcoplasmatic reticulum in muscle cells [31]. Sarcalumenin knock-out mice have an enhanced fatigue resistance [31], suggesting that the expression of the gene is involved in the muscle fatigue mechanism.

Mitochondrial dynamics Excessive exercise increases the muscle atrophy marker atrogin-1 and MuRF-1 mRNA, which is accompanied by increased autophagy and fission of mitochondria in muscle cells [13]. Excessive exercise also decreases peroxisome proliferator-activated receptor (PPAR)- $\gamma$ coactivator PGC-1alpha and complex-I expression. Furthermore, excessive exercise activates JNK and Erh1/2 pathways resulting in induction of p53, p21, and MnSOD expression [13]. These changes in mitochondrial dynamic remodelling may be responsible for muscle damage and damage of immune functions via downregulation of mitochondrial biogenesis and upregulation of autophagy [13].

Other potential mechanisms of EIMUF Other potential factors contributing to the development of EIMUF may be depletion of muscle proteins into the blood [32], reduction of blood and plasma volume (dehydration) as occurring after a marathon [14], acidosis, accumulation of ammonia, elevated serotonin, hypoxia, hyperkalemia, or changes of the genetic profile, particularly in longduration exercise [10]. For example, EIMUF and reduced endurance may be due to reduced expression of the transcriptional coactivator PPAR- $\alpha$ (PGC- $\alpha)$, of which ectopic expression results in increased number and function of mitochondria and increase in oxidative, fatigue-resistant muscle fibers [33]. In patients with chronic obstructive lung disease (COPD) accumulation of plasma ammonia under conditions of metabolic stress during exercise contributes to muscle fatigue, independent of the ventilatory limitation [34]. In a study of tumour suppressor factor p53 knock-out mice it has been shown that p53 initiates mitochondrial biogenesis in response to contractile muscle activity [35].

\section{Postactivation potentiation (PAP)}

When discussing peak-force loss after exhaustion it is important to include the phenomenon of PAP [36]. PAP is characterised by enhancement of exercise performance and maintenance of power output after exercise [36, 37]. The physiologic background of PAP is poorly understood. In a study of 22 endurance-athletes undergoing the University of Montreal track test (UMTT), countermovement jump (CMJ) height improved, peak-power incremented, and athletes maintained their $20 \mathrm{~m}$ sprint performance after exhaustion [36]. In athletes who did not develop PAP, reduction of peak-force, vertical stiffness, and higher vertical displacement of the center of mass correlated with serum lactate levels [36]. PAP can be also observed after other types of exercise, such as rebound throws [37].

\section{Biomarkers of EIMUF}

Studying BMs of EIMUF requires performance of a defined exercise and measurement of parameters (BMs) originating from various different mechanisms of EIMUF. Measurement of BMs may be carried out before, during or after exercise (Fig. 1). Parameters of interest may originate from easily accessible body fluids (wet BMs) or from measuring muscle force, speed of exercise, electrophysiological recordings, cardiac recordings, kinesiology and kinetics, myo-mechanogram (MMG), or from questionnaires (dry BMs). Measuring $\mathrm{VO}_{2} \max$ and $\mathrm{VO}_{2}$ kinetics during exercise provides the basis for volatile BMs of EIMUF. Not each putative BM of EIMUF can be applied to each type of exercise.

\section{Dry biomarkers}

Dry BMs are least invasive and thus usually the ones most easily determined. Dry BMs include measurement of muscle force (power output) before and after or during a workload, analysis of electrophysiological recordings, assessment of cardiac parameters, and scales or questionnaires.

Power output There are various test assemblies to perform exercise and to assess power output from this workload to determine muscle fatigue.

Direct measurement of muscle force. Measuring muscle force before and after predefined workload is one of the most frequently applied means to measure EIMUF. Loss of power output during exercise may reflect muscle fatigue. Muscle force before and after a given task can be measured in a single muscle, in a group of muscles, or in all muscles. Measuring muscle force may be carried out with dynamometers, power 
output measuring devices (probes), clinical assessment of muscle force, or more globally with questionnaires. Various power output measuring devices are available. Various types of exercise may precede force measurement [3]. Leg and arm force production is reduced after any exercise but particularly after a marathon [14]. Maximum isometric force-generating capacity (MIFGC) is reduced immediately after eccentric contraction [38]. MIFGC remains depressed $48 \mathrm{~h}$ after exercise [38]. External/internal rotation isokinetic torque [39] is reduced after exercise, Power output is also reduced after fatiguing leg press exercise [40]. In this test, power is calculated as the instantaneous product of displacement velocity and applied force, whereas work output is calculated as vertical displacement of the weight plates times applied force [40]. Even peak power, however, may show diurnal fluctuations with higher values in the evening compared to the morning [21]. Force of bulbar muscles in children can be assessed by means of the slurp test [41].

Tongue pressure. An example of assessing power output of bulbar muscles is by measuring the tongue pressure. In neuromuscular disorders with bulbar involvement, such as myasthenia, amyotrophic lateral sclerosis (ALS), bulbo-spinal muscular atrophy (SBMA), oculopharyngeal muscular dystrophy (OPMD), myotonic dystrophy type 1 (MD1), or mitochondrial disorders (MIDs), assessment of bulbar functions is mandatory. In a study of 47 patients with SBMA tongue pressure was measured by means of an intra-aortic pressure probe and questionnaires which assessed swallowing [42]. Tongue pressure was reduced in SBMA patients within 3y after disease onset. Tongue pressure was reduced even in patients without dysphagia and repetition of swallowing compensated for tongue weakness in these patients [42]. Tongue pressure was positively correlated with bulbar-related functional scales [42]. Tongue pressure more strongly correlated with muscle strength of the pharyngeal, neck, and upper limb muscles than with lower limb muscles. Though tongue pressure is not validated as a BM of focal EIMUF, it appears a promising monitor of focal EIMUF and weakness of bulbar muscles since power output can be measured over time before and after exercise and correlates well with scales assessing swallowing [42].

Jumping. A further possibility to assess the power output is by means of jumping tests. Most frequently applied is the CMJ test which collects and analysis parameters such as mean power, peak velocity, peak force (PF), peak power (PP), peak torque, jump height, flight time, contact time, and rate of force development (RFD) $[3,43,44]$. The test induces only a minimal amount of additional fatigue itself and thus is useful to monitor EIMUF when carried out before and after exercise $[3,45]$. After an isokinetic, concentric exercise peak torque and RFD decreased even $24 \mathrm{~h}$ after exercise [43].
Jumping performance seems to deteriorate for as long as $72 \mathrm{~h}$ post-exercise whereas strength remains unchanged after an acute bout of intense polymetric exercise [46]. Other jumping tests for measuring the power output include the static jump (SJ) test [46], the vertical jump test [47], and the drop jump [47].

Cycling. Another possibility to assess the power output is by means of cycling on an ergometer. Most commonly applied is the cycle ergometer sprint test which specifically quantifies the concentric component of the fatigueinduced decrement of force production in muscle, which may be overlooked by the CMJ test [48]. The cycle ergometer sprint test is a method of monitoring EIMUF in endurance or power-team-sport athletes [48].

Walking. Walking test represent a frequently applied means to assess the power output. Examples of walking tests are the $6 \mathrm{~m}$ walking test, the $400 \mathrm{~m}$ walking speed test, the timed-up-and-go test [49], the 12-min self-paced walking test, and stair climbing [49].

Running. Another possibility to assess the power output is by measuring running speed or distance. Reduction of running speed is a simple means of indirectly measuring EIMUF but only a global parameter since assessment may also include central fatigue. Decrease of running speed can be particularly observed in longdistance runners (marathoners) [14]. In a study of 22 endurance athletes carrying out the UMTT, the maximum running speed test remained unchanged after the UMTT, possibly due to PAP [36]. Other running tests include the maximum running speed over a distance of $20 \mathrm{~m}$ (20 m sprint test) [36, 47], the total high-intensity running distance (THIR) [45], and the repeated sprint ability (RSA) exercise [50]. Most of the power output tests show diurnal fluctuations with higher values in the afternoon compared to the morning [50].

Electrophysiological BMs Surface EMG analysis. Applying surface-EMG, an interference pattern is recorded by bipolar surface electrodes positioned over the endplate zone of the muscle of interest [51]. Signals are then passed to an A/D converter, are band-pass filtered, and converted to root mean square (RMS) or mean rectified voltage for EMG amplitude, which is roughly equivalent to the mean rectified value (MRV). Additionally, surface EMG interference patterns may be analysed by fast Fourier transformation for median frequency, mean power output, or mean frequency (MF) over a distinct epoch $[8,52]$. In a study of 12 healthy males performing standardised exercise with two different loadings on a knee extension device, post-loading EMG-amplitude was either reduced or increased depending on the type of loading [8]. MRV or RMS are typically increased after exercise. MF and mean power output are typically reduced after exercise [8]. Muscle fatigue is thus characterised by an increase 
in the EMG interference-pattern amplitude (recruitment of additional motor units, increase in firing frequency, synchronisation of discharges) and a shift of the spectrum to the left $[53,54]$. Recovery from muscle fatigue is characterised by a decrease of the EMG-amplitude and a shift of the spectrum to the right [53].

Muscle torque. To assess muscle torque, muscle stimulation is performed on the resting muscle via selfadhesive surface electrodes by delivering single rectangular pulses by a constant-current stimulator to the supplying nerve until a torque plateau is observed [8]. Antagonist muscles are not stimulated. The parameters of interest include the maximum isometric torque, the maximum twitch torque (peak torque), and the half-relaxation time [8]. After exercise the peak torque is typically reduced using variable resistance loadings [8]. Reduction of maximum twitch torque is more pronounced in young as compared to old subjects [8].

M-wave duration. A further electrophysiological method to monitor EIMUF is the assessment of the Mwave after stimulation of motor nerves. Stimulation electrodes (cathodes) are placed such that a weak stimulation current gives the strongest response [8]. Then the stimulating current is increased in $10 \mathrm{~mA}$ steps until a clear plateau in the $\mathrm{M}$-wave amplitude is reached. Thereafter an additional $25 \%$ of stimulation current is applied (supramaximal stimulation) [8]. In a study of 12 healthy males performing standardised exercise on a knee extension device, post-loading peak-to-peak Mwave duration was significantly increased while area and amplitude were decreased after the bout [8].

Conduction velocity. In some studies EIMUF resulted in reduction of the nerve conduction velocity, irrespective if the proband carried out eccentric or concentric exercise [55]. In power athletes as well as in endurance athletes the degree of EIMUF correlated negatively with the nerve conduction velocity [52]. Other studies, however, did not confirm these findings [56]. Contrary to nerve conduction velocity, muscle fiber conduction velocity (MFCV) as assessed by surface EMG decreased with EIMUF at least during dynamic exercise [23, 57, 58]. During static exercise by means of fatiguing isometric contractions, on the contrary, MFCV remained unchanged [58].

Transcranial magnetic stimulation (TMS). In 8 patients with electrical injury undergoing a 2 min exercise with MVC, TMS revealed prolongation of the silent period and an increase of the area and amplitude of the M-wave response [59]. These effects could be increased if patients were exposed to muscle ischemia induced by a blood pressure cuff to simulate EIMUF [59]. The technique allows differentiation between the central and peripheral contribution to EIMUF [59].

EMG fatigue threshold. The EMG fatigue threshold is defined as the exercise intensity an individual can maintain indefinitely without the need to recruit additional motor units, which is associated with an increase in the amplitude of the interference pattern [60]. Recently, a new practical and reliable method to determine the EMG fatigue threshold has been introduced [60]. The physical working capacity at fatigue threshold (PWCFT) is defined as average of the highest power output that results in a non-significant slope coefficient for the EMG amplitude vs. time relationship and the lowest power output that results in a significant positive slope coefficient [61]. Short term exercise increases the fatigue threshold [62]. By means of the PWCFT heavy from severe domains of exercise intensity can be differentiated [63]. Helpful in this respect are also the power output associated with the gas exchange threshold (PGET), the respiratory compensation point (PRCP), and the critical power $[64,65]$. Usually, aerobic exercise training at PWCFT is carried out as a task to produce EIMUF [66]. The absent correlation between PWCFT, PGET, and MPFFT suggests that different physiological mechanisms underlie these three fatigue thresholds [65].

Cardiac parameters. EIMUF is dependent on the muscular blood flow and thus on cardiac function, therefore monitoring and evaluation of basic cardiac parameters can be helpful for assessing EIMUF. Frequently applied cardiac parameters include the heart rate, the postexercise heart-rate recovery (HRR, rate at which heart rate declines after exercise) $[3,45]$, and the heart rate variability (LnrMSSD) calculated from the long-term ECG $[3,45]$. Heart rate is one of the most common parameters to assess the internal load during exercise [3]. This is because of the linear relationship between heart rate and oxygen consumption during steady state exercise [3]. Since there is a positive correlation between THIR, rating of perceived fatigue, and CMJ, HRR and LnrMSSD are promising candidates for non-invasive BMs of the fatigue status in elite soccer players [45].

Movement kinematics and kinetics. Parameters of movement kinematics or kinetics correlated with changes in the muscle fatigue state as measured by EMG interference pattern $[67,68]$. Knee and ankle kinematics can be recorded via optical motion capture [11, 69]. For example, kinematics and kinetics during a CMJ or drop jump can be measured by means of a 9 camera motion analysis system (VICON, $100 \mathrm{~Hz}$ ) and a force plate [47] or the Motion Analysis Corporation 3D kinematic analysis system $(200 \mathrm{~Hz})$ [37]. Evaluated parameters of movement kinematics (jump height, maximal vertical ground reaction force, reactivity strength index, lower limb joint work) may serve as BMs of EIMUF [47]. Other parameters of kinematic measurements by means of motion tracking systems include the mean and variability of joint angles, joint torque, and joint net movements for the shoulder, elbow, and wrist [69]. These parameters usually decrease during fatigue. Increased kinematic 
variability may be found in the more proximal muscles and decreased kinematic variability in the more distal muscles [69]. Kinematic and kinetic adaptations during fatigue are regarded as reactions to reduce biomechanical loading [69].

Myo-mechanogram (MMG). A further tool to monitor EIMUF is the MMG, which measures the parameters peak torque, contraction time, relaxation time, the acceleration force development and relaxation, the slope and tau of force relaxation, and the mean power frequency fatigue threshold $[65,70]$. Peak torque, acceleration of force development, acceleration of relaxation, slope of force relaxation and tau of force relaxation decrease during EIMUF, while contraction time, relaxation time, and the tau of force relaxation increase [70].

Muscle imaging. Functional analysis of muscles can be easily carried out by ultrasound. Particularly muscle fatigue of the diaphragm can be easily monitored by non-invasive ultrasound. A more elaborate method to monitor muscle fatigue is phosphorus magnetic resonance spectroscopy.

Scales and questionnaires. Numerous scores assessing fatigue during or after exercise are available. A major disadvantage of most of these scores is that the CNS component of muscle fatigue is included in the overall assessment of the performance decline after exercise. Further disadvantages are that they rely on subjective information and that many of these scales and questionnaires are not validated for the different types of exercise. Scores most frequently used to assess EIMUF are the perceived rating of fatigue scale [45], the Borg rating perceived exertion (RPE) scale [3], and the session rating of RPE (RPE multiplied by exercise duration). Others include the delayed-onset muscle soreness proto$\mathrm{col}$, the delayed onset muscle soreness (DOMS) score [43], and the Wingate test using the fatigue index [21]. Disadvantage of the RPE is that it shows diurnal fluctuations with higher values at $17.00 \mathrm{~h}$ compared to $7.00 \mathrm{~h}$ [50]. A further disadvantage of the RPE is that it is increased during submaximal tasks due to compensatory higher central and peripheral inputs [10]. Additionally, physical and subjective changes in performance are less severe in real sport activities as compared to simulated activity [10]. Other dry BMs could be psychomotor speed, monitoring of sleep quality, or the training impulse (TRIMP) [3].

\section{Wet biomarkers}

Most of the wet BMs of EIMUF derive from blood and only some from the saliva or urine. The most well-known wet BMs are those originating from ATP depletion, from oxidative stress, from muscle damage, or from immunological compromise [71]. A new candidate of wet BMs is the C-terminal agrin fragment. Recently, it became feasible to measure wet BMs directly in the saliva of probands by application of mass spectroscopy [72].

ATP metabolism Depletion of ATP or increase of adenosine diphosphate (ADP) may contribute to EIMUF [2]. The most well-known BMs of EIMUF originating from ATP metabolism include lactate, ammonia, and oxipurines [2].

Lactate. In case the oxidative phosphorylation fails to provide enough ATP for physiologic requirements, generation of ATP shifts from aerobic processes to anaerobic glycolysis or glycogenolysis [73]. A side product of the anaerobic pathway is lactate. Lactate linearly increases with increasing exercise [8]. However, this linear relation is only maintained below the lactate threshold of about $4 \mathrm{mmol} / \mathrm{l}$ [74]. Lactate threshold is defined as percentage of maximal workload at which lactate increases above normal. Fatigue develops not earlier than above the lactate threshold due to exponential increase of lactate above $4 \mathrm{mmol} / \mathrm{l}$. A disadvantage of serum lactate is that the relation between fatigue and serum lactate becomes nonlinear above the lactate threshold [74]. Further limitations of lactate measurement are that it depends on the ambient temperature, hydration status, diet, lactate clearance rate, glycogen content, previous exercise, and the amount of muscle mass involved in a given exercise [3]. In a study of 12 endurance athletes and 6 power athletes performing fatiguing isokinetic knee flexions/extensions, serum lactate increased with fatigue [52]. Lactate also increased during fatiguing leg press exercise [39]. In a study of 12 healthy subjects, on the contrary, serum lactate decreased after core stabilisation exercise, suggesting that lactate clearance was improved under this condition [75].

Oxipurines (hypoxanthine, xanthine). Oxipurines derive from the degradation of purine nucleotides (adenine, guanine). Oxipurines increase with exercise and are positively correlated with ATP consumption [2]. Oxipurines are a specific and sensitive BM of muscle-cell energy exhaustion during strenuous physical exercise [76]. In a study of 20 healthy males performing isokinetic exercise in a concentric-concentric mode, plasma concentrations of hypoxanthine increased at the end of the exercise [77]. Thus, hypoxanthine was proposed to serve as a BM of work-load effectiveness and of metabolic stress consequences in the muscle [77].

Ammonia. The production of ammonia is triggered by shortage of ATP. In this case, ATP is produced from fusion of two ADPs resulting in one ATP and one AMP. Ammonia originates from the degradation of AMP to IMP and ammonia [2]. Serum ammonia depends on sex. Ammonia increases after short and long duration exercise [2].

Increased oxidative stress BMs of oxidative stress may reflect an increased production of ROS or a decrease of the anti-oxidative capacity. Increased production of ROS 
results in increased lipid peroxidation or increased protein oxidation. The most well-known BM reflecting lipid peroxidation is TBARS. The most well-known BMs reflecting decreased antioxidative capacity include glutathione (GSH) and total antioxidant capacity (TAC).

Thiobarbituric acid reactive substances (TBARS). TBARS are endproducts of lipid peroxidation, which react with thiobarbituric acid to form a fluorescent red adduct $[2,20]$. TBARS are indicators of lipid peroxidation and oxidative stress [2]. TBARS increase with age, physical fitness, and are lower in females as compared to males [2]. TBARS increase $5 \mathrm{~min}$ after starting an incremental cycling exercise but increase also 2 days after exercise with maximal strength attributed to macrophage infiltrationand xanthine-oxidase activation-triggered reperfusion of the ischemic muscle [2]. Increase of TBARS during exercise is accompanied by reduced heat shock protein (HSP) production, suggesting that oxidative stress during exercise results from insufficient production of HSP [78].

Glutathione (GSH). GSH is present in nearly all cells but can be also found in blood and the saliva [2]. GSH is one of the most important physiological antioxidants but has other functions as well [20]. As an antioxidant GSH substitutes halogen-, sulphate-, sulphonate-, phosphate- or nitrate groups. GSH levels decrease with age and are higher in females as compared to males. GSH decreases during high-volume training in healthy subjects, which is correlated with a drop in performance. The maximal decrease of GSH during exercise can be observed approximately $5 \mathrm{~min}$ after starting exercising [2].

Increased oxidative stress TAC comprises the entire pool of specific and non-specific antioxidants within a cell [20]. TAC includes anti-oxidative enzymes (glutathione peroxidase (GPX), catalase, superoxide dismutase (SOD)), non-specific antioxidants (GSH, ascorbic acid, albumin, uric acid, tocopheroles, carotinoids, coenzyme-Q, bilirubine, and the amino acids cysteine, methionine, tyrosine), and metal chelates. TAC is age- and sex-dependent. TAC is higher in the morning compared to the afternoon [50]. TAC usually decreases during exercise.

Other oxidative stress BMs. Other potential BMs of oxidative stress include catalase [20], protein carbonyls, SOD, isoprostanes, malondialdehyde, uric acid, total bilirubine, and GPX. They have been described in detail elsewhere [2]. A disadvantage of most oxidative stress BMs is that they show diurnal fluctuations with lower values in the evening compared to the morning [50]. Antioxidant parameters at rest are higher in the morning as compared to the evening [21]. Antioxidative efficiency decreases with age and damage from oxidative stress increases with age [71].

Inflammatory response It is well appreciated that particularly long-duration exercise triggers an inflammatory response in the fatiguing muscle. The most frequently applied BMs of this inflammatory response include the leukocyte count, cortisol, and interleukin-6 (IL-6).

Leukocytes. After a marathon, the leukocyte count is increased by $163 \%$, the thrombocyte count by $20 \%$ and the erythrocyte count by $2.1 \%$ [14]. However, a study on the leukocyte response to bench stepping (high stress) and to repeated eccentric muscle action (low stress) 4, 24,48 , and $72 \mathrm{~h}$ after exercise and by assessing perceived muscle soreness by means of the delayed-onset muscle soreness score showed that systemic stress elicited during an acute bout of eccentric exercise has a stronger influence on the functional leukocyte response than the degree of muscle damage induced [79].

Cortisol. Cortisol levels increase after various types of exercise. Salivary free cortisol increases in middle and long-distance runners after the race [80]. Salivary free cortisol correlates negatively with $\mathrm{CMJ}$ height after middle-distance run [80].

Interleukin-6 (IL-6). IL-6 is secreted by T-lymphocytes and macrophages to stimulate the immune response after trauma or other tissue damage, leading to inflammation [2]. IL-6 also functions as a myokine, such that it increases in response to muscle contraction [81, 82]. IL-6 mobilises substrates, increases uptake of glucose, hepatic glucose production during exercise, insulin-mediated glucose disposal or lipolysis, and fat oxidation [2]. IL-6 is higher in females as compared to males. IL- 6 increases exponentially with exercise. IL-6 is released to the circulation shortly after onset of exercise, precedes the appearance of other cytokines, peaks immediately after exercise, and returns to normal within a few hours after the bout [83]. IL-6 correlates with duration, intensity, number of active muscles, and endurance capacity [2].

Other inflammatory BMs. Other potential inflammatory markers to monitor EIMUF include C-reactive protein [46], TNF-alpha, and other interleukines (e.g. IL-1b, IL-10).

Muscle damage BMs of muscle damage include serum $\mathrm{CK}, \mathrm{LDH}$ and myoglobin. Additionally, myoglobin can be determined in the urine. These BMs are particularly increased after middle- or long-duration exercise, and are hardly useful for monitoring short-duration exercise. CK and LDH remain increased even 24-72 h after 4 sets of 10 repetition maximum loads for the chest press, pullover, biceps curl, triceps extension, leg extension, and prone leg curl [84]. Neuromuscular function is also compromised for up to $48 \mathrm{~h}$ after a Rugby match as evidenced by decreased peak RFD, PP, and PF on the CMJ [44]. CK increased 30 min after a rugby match with a maximum $24 \mathrm{~h}$ later and remaining elevated for $120 \mathrm{~h}$ [44]. BMs of muscle damage show diurnal fluctuations with higher values in the evening compared to the morning [50]. Damage to muscle tissue after a Rugby League match persists for at least 5 days 
post-match [44]. Disadvantage of CK is that the temporal relationship with muscle recovery is poor [3].

C-terminal agrin fragment (CAF) Agrin is a protein of the neuromuscular junction. Its activity is regulated by neurotrypsin, which cleaves it into the C-terminal agrin fragment (CAF). If there is excessive neurotrypsin activity degeneration of the neuromuscular junction and thus decreased power output ensues [61]. In a study of 22 healthy older subjects the CAF was determined from blood [61]. Additionally, EMG-signals were recorded at PWCFT during a 2 min discontinuous incremental cycle ergometer exercise $[85,86]$. Recorded EMG signals were converted as RMS. In men there was a negative correlation between CAF and PWCFT [61]. It was concluded that CAF concentrations are related to onset of muscle fatigue (only in men) irrespective of age and BMI [61].

Other potential wet BMs Other potential BMs for monitoring EIMUF include testosterone, salivary immunoglobulinA, natural killer cell activity, neutrophil phagocytic activity, methyl-histidine, glucose-1-phosphate, glucose-6-phosphate, and taurine, of which some can be easily measured in the saliva by means of capillary electrophoresis and time-of-flight mass spectroscopy (CE-TOFMS) [46, 72].

\section{Volatile BMs}

To sustain endurance performance of the muscle the ability to deliver oxygen to the muscle and the ability of the muscle to utilise a given oxygen load for a sustained period of time (respiratory exchange rate) are important [87]. Maximal oxygen uptake $\left(\mathrm{VO}_{2} \max \right)$ as measured by breath-by-breath open-circuit spirometry is thus a well-established parameter to assess muscle performance [88]. VO2 max decreases with EIMUF but decreases also during age [89]. $\mathrm{VO}_{2}$ max is defined as highest $\mathrm{VO}_{2}$ value when at least 2 of 3 criteria are met: 1 . a plateau of the heart rate or a heart rate within $10 \%$ of the age-predicted maximal heart rate, 2. A plateau of $\mathrm{VO}_{2}$, or 3. A respiratory exchange rate $>1$ [88]. Ventilatory threshold is defined as intersection of 2 regression lines $\left(\mathrm{V}_{\mathrm{E}}\right.$ and $\left.\mathrm{VO}_{2}\right)$ [88]. Recently, it has been proposed that slow $\mathrm{VO}_{2}$ kinetics should be regarded as a volatile $\mathrm{BM}$ of exercise intolerance [90]. Slow $\mathrm{VO}_{2}$ kinetics after exercise is correlated with greater $\mathrm{PCr}$ decrease in the cytoplasm [90]. Conversely, small $\mathrm{PCr}$ decrease is strictly correlated with fast $\mathrm{VO}_{2}$ kinetics [90]. Putative volatile BMs of EIMUF other than $\mathrm{VO}_{2}$ could be the $\mathrm{VCO}_{2}$, and the respiratory exchange ratio.

\section{Combinations of BMs}

Two or more wet BMs may be combined or two or more dry BMs may be combined for monitoring EIMUF. It is also possible to mix wet, volatile, and dry BMs in a variable ratio of components.
Real-time fatigue monitoring system Recently, a realtime fatigue monitoring system has been introduced which quantifies EIMUF during cycling at a constant speed of 60 RPM [91]. The system was equipped with a fatigue progression measure, which synchronously recorded surface EMG signals of the lateral vastus and gastrocnemius muscles in one leg as well as cycling speed in a real-time mode [91]. Additionally, cycling velocity, cycling time, kinesiological data, heart rate and Borg RPE scale values were taken each minute [91]. From these measures the fatigue progression measure was calculated to measure onset-time and progression of EIMUF [91]. The online fatigue monitoring system was validated in healthy subjects and met the intended purposes.

Fatiguing leg press exercise Static fatiguing leg press exercise until exhaustion resulted in increased blood lactate and increased MRV of the agonist and antagonist muscles [39]. Simultaneously, mean power output and MF of surface-EMG decreased in agonist and antagonist muscles [39]. Shift to lower frequencies, increase of $\mathrm{MRV}$, and accumulation of lactate were regarded as independent BMs of fatigue [39].

\section{Discussion}

This review shows that there is progress in the development of BMs to monitor EIMUF, that there are different types of EIMUF depending on the individual, type, intensity, and duration of exercise, and the environmental conditions, that there is a huge number of putative wet, volatile, and dry BMs to monitor EIMUF, and that there is controversy if a single $\mathrm{BM}$ or multiple $\mathrm{BMs}$ are more suitable for monitoring EIMUF [1]. This review also shows that EIMUF is due to different simultaneously active mechanisms and that the amount of contribution of these different mechanisms changes with type, intensity, and duration of exercise. The choice of a particular BM may additionally be influenced by sex, ethnicity, climate, and daytime. Additionally, only few BMs have strong scientific evidence supporting their use, and there is yet to be a single definite BM of EIMUF described in the literature [3]. A single definite tool of BMs that is accurate and reliable is not currently evident [3]. Currently, there is no gold standard for monitoring the various physiological alterations arising in the skeletal muscle during EIMUF. This is why we recommend to analyse several BMs simultaneously and to further evaluate known BMs and to search intensively for new BMs. Future work should be particularly directed towards the question which combination of BMs is most suitable to monitor EIMUF from a specific type of exercise in a specific group of probands.

Disadvantages of most BMs currently available are that appropriate reference limits are available only for a single given task and that BMs are frequently not validated in 
general and particularly for other tasks. Since reference limits for BMs for physically active subjects or athletes are lacking, there is a need to establish such reference limits adapted to different types of exercise [1]. Each BM needs to be validated for each type of exercise, to find out which pattern of BMs is most suitable for monitoring EIMUF during or after a particular exercise mode. Additionally, the effectivity of a particular BM for monitoring muscle fatigue needs to be investigated and validated for all types of exercise. If a BM is sufficiently validated, it can be used for monitoring performance and progress in training, identifying overtraining, health-related aspects, which can be modified by regular physical activity, or the physiological response to exercise in healthy and diseased subjects, professional athletes, or amateur athletes. In sport medicine BMs may be helpful to identify subjects who are at an increased risk of poor adaptation to training.

The choice of BMs for monitoring EIMUF is much dependent on the type of exercise to be monitored. Static exercise allows applying more complex and invasive BMs also directly during the workout whereas dynamic exercise allows only determining and monitoring online core temperature, heart rate, and speed. EIMUF from dynamic exercise can be more easily monitored by tests carried out before and after the workload. As an example, electrophysiological techniques are hardly applicable during dynamic exercise and remain the domain of static exercise induced fatigue. Wet BMs also remain the domain of static exercise with determination before and after the bout since measurement during dynamic exercise is only possible by interrupting the task for collecting the tissue of interest. The most intensively investigated and most frequently applied BMs of EIMUF are wet BMs, which include markers of ATP depletion, oxidative stress, of muscle damage, and inflammatory markers. Increasingly investigated are also dry BMs since they are usually more easily obtained and less invasive. Other factors that determine which BMs should be used in a specific study derive from the examiner's end ( availability of special equipment, the expertise of the researchers in using it, how time-consuming the technique is) and from the examined individuals' end ( ability to perform a specific task with a specific disease or in a specific age group).

\section{Conclusions}

This review shows that some well-known BMs are available and promising BMs have been recently introduced to monitor EIMUF although the pathophysiology of EIMUF remains to be elucidated. To apply a single BM or a combination of BMs for monitoring EIMUF it is essential that their reliability and applicability is validated in appropriate studies on healthy and diseased, trained and untrained, old and young subjects using all different types of exercise. Currently, it appears that a combination of BMs reflects the various different fatigue mechanisms during a certain exercise more adequately than a single $\mathrm{BM}$. The most appropriate sets of BMs for a given task, however, remain to be ascertained and to achieve scientific legitimacy.

\section{Abbreviations \\ ADP: Adenosin-diphosphate; ALS: Amyotrophic lateral sclerosis; AMP: Adenosin-monophosphate; ATP: Adenosin-triphosphate; BM: Biomarker; CAF: C-terminal agrin fragment; CE-TOFMS: Capillary electrophoresis and time-of-flight mass spectroscopy; CK: Creatine-kinase; CMJ: Counter- movement jump; CNS: Central nervous system; COPD: Chronic obstructive pulmonary disease; CSI: Cardiac stress index; DFA: Detrended fluctuation analysis; DOMS: Delayed onset muscle soreness; EIMUF: Exercise-induced muscle fatigue; EMG: Electromyography; GPX: Glutathione peroxidase; GSH: Glutathione; HRR: Heart-rate recovery; HSP: Heat shock protein; IL: Interleukine; IMP: Inosine monophosphate; LDH: Lactate dehydrogenase; LnrMSSD: Heart rate variability; MD1: Myotonic dystrophy 1; MF: Mean frequency; MFCV: Muscle fiber conduction velocity; MID: Mitochondrial disorder; MIFGC: Maximum isometric force-generating capacity; MMG: Myo- mechanogram; MRV: Mean rectified voltage; MVC: Maximal voluntary contraction; OPMD: Oculo-pharyngeal muscular dystrophy; PAP: Postactivation potentiation; PF: Peak force; PGC: Transcriptional coactivator peroxisome-proliferator-activated receptor alpha; PGET: Power output associated with the gas exchange threshold; PP: Peak power; PPAR: Peroxisome proliferator-activated receptor; PRCP: Respiratory compensation point; PRF: Perceived rating of fatigue; PWCFT: Physical working capacity at fatigue threshold; RFD: Rate of force development; RMS: Root mean square; ROS: Reactive oxygen species; RPE: Rating perceived exertions; RSA: Repeated sprint ability; SBMA: Spino-bulbar muscular atrophy; SOD: Superoxide dismutase; TAC: Total antioxidant capacity; \\ TBARS: Thiobarbituric acid reactive substances; THIR: Total high-intensity run- ning distance; TMS: Transcranial magnetic stimulation; TRIMP: Training impulse; UMTT: University of Montreal track test.}

\section{Competing interests}

The authors declare that they have no competing interests.

\section{Authors' contribution}

JF conceptualised and designed the review and drafted the manuscript. VD participated in the design and coordination and helped to draft the manuscript. Both authors read and approved the final manuscript.

\section{Author details}

${ }^{1}$ Krankenanstalt Rudolfstiftung, Postfach 20, 1180 Vienna, Austria. ${ }^{2}$ Medical Center, Tel-Aviv, Israel.

Received: 8 October 2015 Accepted: 5 January 2016

Published online: 21 January 2016

\section{References}

1. Palacios G, Pedrero-Chamizo R, Palacios N, Maroto-Sánchez B, Aznar S, González-Gross M, et al. Biomarkers of physical activity and exercise. Nutr Hosp. 2015;31 Suppl 3:237-44.

2. Finsterer J. Biomarkers of peripheral muscle fatigue during exercise. BMC Musculoskeletal Dis. 2012;13:218. http://www.biomedcentral.com/ $1471-2474 / 13 / 218$

3. Halson SL. Monitoring training load to understand fatigue in athletes. Sports Med. 2014;44 suppl 2:S139-47. doi:10.1007/s40279-014-0253-z.

4. Skurvydas A, Streckis V, Mickeviciene D, Kamandulis S, Stanislovaitis A, Mamkus G. Effect of age on metabolic fatigue and on indirect symptoms of skeletal muscle damage after stretch-shortening exercise. J Sports Med Phys Fitness. 2006;46:431-41.

5. Sawka MN, Burke LM, Eichner ER, Maughan RJ, Montain SJ, Stachenfeld NS, et al. American College of Sports Medicine position stand. Exercise and fluid replacement. Med Sci Sports Exerc. 2007:39:377-90.

6. Pennuto M, Greensmith L, Pradat PF, Sorarù G; European SBMA Consortium. 210th ENMC International Workshop: Research and clinical management of patients with spinal and bulbar muscular atrophy, 27-29 March, 2015, 
Naarden, The Netherlands. Neuromuscul Disord. 2015. doi:10.1016/j.nmd. 2015.06.462.

7. Steinberg JG, Delliaux S, Jammes Y. Reliability of different blood indices to explore the oxidative stress in response to maximal cycling and static exercises. Clin Physiol Funct Imaging. 2006;26:106-12.

8. Walker S, Peltonen $\mathrm{H}$, Avela J, Häkkinen K. Neuromuscular fatique in young and older men using constant or variable resistance. Eur J Appl Physiol. 2013;113:1069-79.

9. Edwards RHT. Biochemical basis of fatigue in exercise performance. Champaign: Human Kinetics; 1983.

10. Knicker AJ, Renshaw I, Oldham AR, Cairns SP. Interactive processes link the multiple symptoms of fatigue in sport competition. Sports Med. 2011;41:307-28.

11. Nessler JA, Huynh H, McDougal M. A single bout of resistance exercise does not affect nonlinear dynamics of lower extremity kinematics during treadmill walking. Gait Posture. 2011;34:285-7.

12. Garvican LA, Hammond K, Varley MC, Gore CJ, Billaut F, Aughey RJ. Lower running performance and exacerbated fatigue in soccer played at $1600 \mathrm{~m}$ Int J Sports Physiol Perform. 2014;9:397-404.

13. Feng Z, Bai L, Yan J, Li Y, Shen W, Wang Y, et al. Mitochondrial dynamic remodeling in strenuous exercise-induced muscle and mitochondrial dysfunction: regulatory effects of hydroxytyrosol. Free Radic Biol Med. 2011;50:1437-46.

14. Abián-Vicen J, Salinero JJ, González-Millán C, Areces F, Ruiz D, Gallo C, et al. Running pace decrease during a marathon is positively related to blood markers of muscle damage. PLoS One. 2013;8:e57602. doi:10.1371/journal. pone.0057602.

15. Rzanny R, Grassme R, Reichenbach JR, Scholle HC, Kaiser WA. Investigations of back muscle fatigue by simultaneous 31P MRS and surface EMG measurements. Biomed Tech (Berl). 2006;51:305-13.

16. Sherman WM, Costill DL, Fink WJ, Hagerman FC, Armstrong LE, Murray TF. Effect of a $42.2-\mathrm{km}$ footrace and subsequent rest or exercise on muscle glycogen and enzymes. J Appl Physiol Respir Environ Exerc Physiol. 1983;55:1219-24.

17. Callow M, Morton A, Guppy M. Marathon fatigue: the role of plasma fatty acids, muscle glycogen and blood glucose. Eur J Appl Physiol Occup Physiol. 1986;55:654-61.

18. Vissing J, Haller RG. Mechanisms of exertional fatique in muscle glycogenoses. Neuromuscul Disord. 2012;22 suppl 3:S168-71.

19. Samaras A, Tsarouhas K, Paschalidis E, Giamouzis G, Triposkiadis F, Tsitsimpikou C, et al. Effect of a special carbohydrate-protein bar and tomato juice supplementation on oxidative stress markers and vascular endothelial dynamics in ultra-marathon runners. Food Chem Toxicol. 2014 69:231-6. doi:10.1016/j.fct.2014.03.029

20. Kerasioti E, Kiskini A, Veskoukis A, Jamurtas A, Tsitsimpikou C, Tsatsakis AM, et al. Effect of a special carbohydrate-protein cake on oxidative stress markers after exhaustive cycling in humans. Food Chem Toxicol. 2012;50:2805-10.

21. Hammouda O, Chtourou H, Chahed H, Ferchichi S, Chaouachi A, Kallel C, et al. High intensity exercise affects diurnal variation of some biological markers in trained subjects. Int J Sports Med. 2012;33:886-91.

22. Peake JM, Suzuki K, Coombes JS. The influence of antioxidant supplementation on markers of inflammation and the relationship to oxidative stress after exercise. J Nutr Biochem. 2007:18:357-71.

23. Zwarts MJ, Bleijenberg G, van Engelen BG. Clinical neurophysiology of fatigue. Clin Neurophysiol. 2008;119:2-10.

24. Fortune $E$, Lowery MM. Effect of extracellular potassium accumulation on muscle fiber conduction velocity: a simulation study. Ann Biomed Eng. 2009;37:2105-17

25. Wasserman K, Stringer WW, Casaburi R, Zhang YY. Mechanism of the exercise hyperkalemia: an alternate hypothesis. J Appl Physiol. 1997;83:631-43.

26. Del Coso J, González-Millán C, Salinero JJ, Abián-Vicén J, Soriano L, Garde S, et al. Muscle damage and its relationship with muscle fatigue during a halfiron triathlon. PLoS One. 2012;7(8):e43280. doi:10.1371/journal.pone.0043280.

27. Del Coso J, Salinero JJ, Abián-Vicen J, González-Millán C, Garde S, Vega P, et al. Influence of body mass loss and myoglobinuria on the development of muscle fatique after a marathon in a warm environment. Appl Physiol Nutr Metab. 2013;38:286-91

28. Hikida RS, Staron RS, Hagerman FC, Sherman WM, Costill DL. Muscle fiber necrosis associated with human marathon runners. J Neurol Sci. 1983;59: 185-203.

29. Chen SW, Liaw JW, Chang YJ, Chan HL, Chiu LY. A cycling movement based system for real-time muscle fatigue and cardiac stress monitoring and analysis. PLoS One. 2015;10(6):e0130798. doi:10.1371/journal.pone.0130798.
30. Behnke BJ, Delp MD, McDonough P, Spier SA, Poole DC, Musch TI. Effects of chronic heart failure on microvascular oxygen exchange dynamics in muscles of contrasting fiber type. Cardiovasc Res. 2004;61:325-32.

31. Zhao X, Yoshida M, Brotto L, Takeshima H, Weisleder N, Hirata Y, et al. Enhanced resistance to fatigue and altered calcium handling properties of sarcalumenin knockout mice. Physiol Genomics. 2005;23:72-8.

32. Schiff HB, MacSearraigh ET, Kallmeyer JC. Myoglobinuria, rhabdomyolysis and marathon running. Q J Med. 1978;47:463-72.

33. Handschin C, Chin S, Li P, Liu F, Maratos-Flier E, Lebrasseur NK, et al. Skeletal muscle fiber-type switching, exercise intolerance, and myopathy in PGC1alpha muscle-specific knock-out animals. J Biol Chem. 2007;282:30014-21.

34. Calvert LD, Steiner MC, Morgan MD, Singh SJ. Plasma ammonia response to incremental cycling and walking tests in COPD. Respir Med. 2010;104:675-81.

35. Saleem A, Adhihetty PJ, Hood DA. Role of p53 in mitochondrial biogenesis and apoptosis in skeletal muscle. Physiol Genomics. 2009:37:58-66.

36. Boullosa DA, Tuimil JL, Alegre LM, Iglesias E, Lusquiños F. Concurrent fatigue and potentiation in endurance athletes. Int I Sports Physiol Perform. 2011;6:82-93.

37. Harrison AJ. Throwing and catching movements exhibit post-activation potentiation effects following fatigue. Sports Biomech. 2011;10:185-96.

38. Carson RG, Riek S, Shahbazpour N. Central and peripheral mediation of human force sensation following eccentric or concentric contractions. J Physiol. 2002;539:913-25.

39. Detanico D, Dal Pupo J, Franchini E, Dos Santos SG. Effects of successive judo matches on fatigue and muscle damage markers. J Strength Cond Res. 2015;29:1010-6.

40. Gorostiaga EM, Navarro-Amézqueta I, González-Izal M, Malanda A, Granados C, Ibáñez J, et al. Blood lactate and sEMG at different knee angles during fatiguing leg press exercise. Eur J Appl Physiol. 2012;112:1349-58.

41. Hudspeth MP, Holden KR, Crawford TO. The "slurp" test: bedside evaluation of bulbar muscle fatigue. Pediatrics. 2006;1 18:e530-3.

42. Mano T, Katsuno M, Banno H, Suzuki K, Suga N, Hashizume A, et al. Tongue pressure as a novel biomarker of spinal and bulbar muscular atrophy. Neurology. 2014;82:255-62.

43. Molina R, Denadai BS. Dissociated time course recovery between rate of force development and peak torque after eccentric exercise. Clin Physiol Funct Imaging. 2012;32:179-84

44. McLellan CP, Lovell DI, Gass GC. Markers of postmatch fatigue in professional Rugby League players. J Strength Cond Res. 2011;25:1030-9.

45. Thorpe RT, Strudwick AJ, Buchheit M, Atkinson G, Drust B, Gregson W. Monitoring fatigue during the in-season competitive phase in elite soccer players. Int J Sports Physiol Perform. 2015;(in press).

46. Chatzinikolaou A, Fatouros IG, Gourgoulis V, Avloniti A, Jamurtas AZ, Nikolaidis MG, et al. Time course of changes in performance and inflammatory responses after acute plyometric exercise. J Strength Cond Res. 2010;24:1389-98.

47. Strutzenberger G, Moore J, Griffiths H, Schwameder H, Irwin G. Effects of gluteal kinesio-taping on performance with respect to fatigue in rugby players. Eur J Sport Sci. 2015;3:1-7.

48. Wehbe G, Gabett TJ, Dwyer D, McLellan C, Coad S. Monitoring neuromuscular fatigue in team-sport athletes using a cycle-ergometer test. Int I Sports Physiol Perform. 2015;10:292-7. doi:10.1123/ijspp.2014-0217.

49. Storer TW, Woodhouse L, Magliano L, Singh AB, Dzekov C, Dzekov J, et al. Changes in muscle mass, muscle strength, and power but not physical function are related to testosterone dose in healthy older men. J Am Geriatr Soc. 2008;56:1991-9.

50. Hammouda O, Chtourou H, Chahed H, Ferchichi S, Kallel C, Miled A, et al. Diurnal variations of plasma homocysteine, total antioxidant status, and biological markers of muscle injury during repeated sprint: effect on performance and muscle fatigue-a pilot study. Chronobiol Int. 2011;28:958-67.

51. Hermens HJ, Freriks B, Merletti R, Stegeman D, Blok J, Rau G, et al. European recommendations for surface electromyography, results of the SENIAM project 1999. Enschede: Roessingh Research and Development; 1999. p. 44-6.

52. Rainoldi A, Gazzoni M, Merletti R, Minetto MA. Mechanical and EMG responses of the vastus lateralis and changes in biochemical variables to isokinetic exercise in endurance and power athletes. J Sports Sci. 2008;26:321-31.

53. Cifrek M, Medved V, Tonković S, Ostojić S. Surface EMG based muscle fatigue evaluation in biomechanics. Clin Biomech (Bristol, Avon). 2009;24:327-40.

54. Pereira GR, de Oliveira LF, Nadal J. Isometric fatigue patterns in time and time-frequency domains of triceps surae muscle in different knee positions. J Electromyogr Kinesiol. 2011;21:572-8. 
55. Gonzalez-Izal M, Lusa Cadore E, Izquierdo M. Muscle conduction velocity, surface electromyography variables, and echo intensity during concentric and eccentric fatigue. Muscle Nerve. 2014;49:389-97.

56. Beck TW, Ye X, Wages NP. Local muscle endurance is associated with fatigue-based changes in electromyographic spectral properties, but not with conduction velocity. J Electromyogr Kinesiol. 2015;25:451-6.

57. Stewart D, Farina D, Shen C, Macaluso A. Muscle fibre conduction velocity during a 30-s Wingate anaerobic test. J Electromyogr Kinesiol. 2011;21:418-22.

58. Schmitz JP, van Dijk JP, Hilbers PA, Nicolay K, Jeneson JA, Stegeman DF. Unchanged muscle fiber conduction velocity relates to mild acidosis during exhaustive bicycling. Eur J Appl Physiol. 2012;112:1593-602.

59. Kashigar A, Udupa K, Fish J, Chen R. Neurophysiological assessment of fatigue in electrical injury patients. Exp Brain Res. 2014;232:1013-23.

60. Galen SS, Guffey DR, Coburn JW, Malek MH. Determining the electromyographic fatigue threshold following a single visit exercise test. J Vis Exp. 2015;(101). doi:10.3791/52729.

61. Stout JR, Fragala MS, Hoffman JR, Robinson 4th EH, Mccormack WP, Townsend $J R$, et al. C-terminal agrin fragment is inversely related to neuromuscular fatigue in older men. Muscle Nerve. 2015;51:132-3.

62. Emerson NS, Stout JR, Fukuda DH, Robinson EH, Iv STC, Beyer KS, et al. Resistance training improves capacity to delay neuromuscular fatigue in older adults. Arch Gerontol Geriatr. 2015;61:27-32.

63. Camic CL, Kovacs AJ, Enquist EA, VanDusseldorp TA, Hill EC, Calantoni AM, et al. An electromyographic-based test for estimating neuromuscular fatigue during incremental treadmill running. Physiol Meas. 2014;35:2401-13.

64. Bergstrom HC, Housh TJ, Cochrane KC, Jenkins ND, Lewis RW, Traylor DA, et al. An examination of neuromuscular and metabolic fatigue thresholds. Physiol Meas. 2013;34:1253-67.

65. Zuniga JM, Housh TJ, Camic CL, Hendrix CR, Schmidt RJ, Mielke M, et al. A mechanomyographic fatigue threshold test for cycling. Int J Sports Med. 2010;31:636-43.

66. Jenkins ND, Buckner SL, Baker RB, Bergstrom HC, Cochrane KC, Weir JP, et al. Effects of 6 weeks of aerobic exercise combined with conjugated linoleic acid on the physical working capacity at fatigue threshold. J Strength Cond Res. 2014;28:2127-35.

67. Dingwell JB, Joubert JE, Diefenthaeler F, Trinity JD. Changes in muscle activity and kinematics of highly trained cyclists during fatigue. IEEE Trans Biomed Eng. 2008;55:2666-74.

68. Song M, Segala DB, Dingwell JB, Chelidze D. Slow-time changes in human EMG muscle fatigue states are fully represented in movement kinematics. J Biomech Eng. 2009;131:021004. doi:10.1115/1.3005177.

69. Qin J, Lin JH, Faber GS, Buchholz B, Xu X. Upper extremity kinematic and kinetic adaptations during a fatiguing repetitive task. J Electromyogr Kinesiol. 2014;24:404-11.

70. Cè E, Rampichini S, Limonta E, Esposito F. Torque and mechanomyogram correlations during muscle relaxation: effects of fatigue and time-course of recovery. J Electromyogr Kinesiol. 2013;23:1295-303.

71. Bouzid MA, Hammouda O, Matran R, Robin S, Fabre C. Changes in oxidative stress markers and biological markers of muscle injury with aging at rest and in response to an exhaustive exercise. PLoS One. 2014;9(3):e90420. doi:10.1371/journal.pone.0090420.eCollection2014.

72. Ra SG, Maeda S, Higashino R, Imai T, Miyakawa S. Metabolomics of salivary fatigue markers in soccer players after consecutive games. Appl Physiol Nutr Metab. 2014;39:1120-6.

73. Gosker HR, Schols AM. Fatigued muscles in COPD but no finishing line in sight. Eur Respir J. 2008;31:693-4.

74. Siciliano G, Pasquali L, Manca ML, Del Corona A, Tessa A, Patrono C, et al. The relationship between anaerobic lactate threshold and plasma catecholamines during incremental exercise in hereditary spastic paraplegia. Funct Neurol. 2003;18:83-7.

75. Navalta JW, Hrncir SP. Core stabilization exercises enhance lactate clearance following high-intensity exercise. J Strength Cond Res. 2007;21:1305-9.

76. Bianchi GP, Grossi G, Bargossi AM. May peripheral and central fatigue be correlated? Can we monitor them by means of clinical laboratory tools? J Sports Med Phys Fitness. 1997;37:194-9.

77. Speranza L, Grilli A, Patruno A, Franceschelli S, Felzani G, Pesce M, et al. Plasmatic markers of muscular stress in isokinetic exercise. J Biol Regul Homeost Agents. 2007;21:21-9.

78. Jammes Y, Steinberg JG, Delliaux S, Brégeon F. Chronic fatigue syndrome combines increased exercise-induced oxidative stress and reduced cytokine and Hsp responses. J Intern Med. 2009;266:196-206.
79. Saxton JM, Claxton D, Winter E, Pockley AG. Peripheral blood leucocyte functional responses to acute eccentric exercise in humans are influenced by systemic stress, but not by exercise-induced muscle damage. Clin Sci (Lond). 2003;104:69-77.

80. Balsalobre-Fernández C, Tejero-González CM, del Campo-Vecino J. Relationships between training load, salivary cortisol responses and performance during season training in middle and long distance runners. PLoS One. 2014;9(8): e106066. doi:10.1371/journal.pone.0106066.eCollection2014.

81. Brandt C, Pedersen BK. The role of exercise-induced myokines in muscle homeostasis and the defense against chronic diseases. J Biomed Biotechnol. 2010;(in press)

82. Pedersen BK. IL-6 signaling in exercise and disease. Biochem Soc Trans. 2007:35:1295-7.

83. Febbraio MA, Pedersen BK. Contraction-induced myokine production and release: is skeletal muscle an endocrine organ? Exerc Sport Sci Rev. 2005;33:114-9.

84. Machado M, Koch AJ, Willardson JM, Pereira LS, Cardoso MI, Motta MK, et al. Effect of varying rest intervals between sets of assistance exercises on creatine kinase and lactate dehydrogenase responses. J Strength Cond Res. 2011;25:1339-45.

85. Emerson NS, Fukuda DH, Stout JR, Robinson 4th EH, McCormack WP, Scanlon TC, et al. Physical working capacity at fatigue threshold (PWCFT) is associated with sarcopenia-related body composition and measures of functionality in older adults. Arch Gerontol Geriatr. 2014:59:300-4.

86. deVries HA, Brodowicz GR, Robertson LD, Svoboda MD, Schendel JS, Tichy AM, et al. Estimating physical working capacity and training changes in the elderly at the fatigue threshold (PWCft). Ergonomics. 1989;32:967-77.

87. Snell PG, Mitchell JH. The role of maximal oxygen uptake in exercise performance. Clin Chest Med. 1984;5:51-62.

88. Smith-Ryan AE, Woessner MN, Melvin MN, Wingfield HL, Hackney AC. The effects of beta-alanine supplementation on physical working capacity at heart rate threshold. Clin Physiol Funct Imaging. 2014;34:397-404.

89. Makrides L, Heigenhauser GJ, McCartney N, Jones NL. Maximal short term exercise capacity in healthy subjects aged 15-70 years. Clin Sci (Lond). 1985;69:197-205.

90. Grassi B, Porcelli S, Salvadego D, Zoladz JA. Slow VO2 kinetics during moderate-intensity exercise as markers of lower metabolic stability and lower exercise tolerance. Eur J Appl Physiol. 2011;111:345-55.

91. Chen SW, Liaw JW, Chan HL, Chang YJ, Ku CH. A real-time fatigue monitoring and analysis system for lower extremity muscles with cycling movement. Sensors (Basel). 2014;14:12410-24. doi:10.3390/s140712410.

\section{Submit your next manuscript to BioMed Central and we will help you at every step:}

- We accept pre-submission inquiries

- Our selector tool helps you to find the most relevant journal

- We provide round the clock customer support

- Convenient online submission

- Thorough peer review

- Inclusion in PubMed and all major indexing services

- Maximum visibility for your research

Submit your manuscript at www.biomedcentral.com/submit
Biomed Central 